\title{
Researches of the Dynamics of Cutting Through of the Material by a Water-Ice Jet
}

\author{
Mikhail Burnashov ${ }^{1}$, Anton Tryastsin ${ }^{1}$, Yuriy Stepanov ${ }^{1}$, and Vitaly Kuzin ${ }^{1, *}$ \\ ${ }^{1}$ Orel State University of I.S. Turgenev, Komsomolskaya st. 95, Orel, Russian Federation
}

\begin{abstract}
Hypersonic ice abrasive water jet cutting is a subject of great attention in the production industry. The paper presents some mathematical equations for determining energy parameters of ice water jet cutting. The mathematical model presented in the article can be used for developing a technology for cutting, strengthening, cleaning, or destructing various materials.
\end{abstract}

\section{Introduction}

In recent decades, high-pressure water jet cutters have found the most widespread use for processing explosive materials, disposing of ammunition, and cleaning machine parts in chemical and nuclear industries. It includes cutting of sheet materials, lathe cutting of ammunition, cleaning, etc. There are water-only cutters and abrasive jet cutters with a mixture of water and abrasives such as ice or polymers. The working pressure of a jet is from $150 \mathrm{MPa}$ to $450 \mathrm{MPa}$, and the diameter of nozzles is from $0.2 \mathrm{~mm}$ to $1.5 \mathrm{~mm}$ [1-5].

\section{Dynamic model}

Cutting occurs in materials when the total stress caused by the hydrodynamic pressure of a jet of water and the dynamic effect of the high-speed flow of ice particles, as well as resulting elastic deformation at the cut point exceed certain predetermined values which characterize rupture strength of the cut material:

$$
\sigma_{\mathrm{x}}(t, \phi)+\sigma(\varepsilon)+\sigma_{h p} \geq \sigma_{r s} .
$$

The sooner these values are reached the more efficient the cutting process is.

Deformation at the cut point can be described with a second-order linear differential equation:

$$
y^{\prime \prime}+2 \alpha y^{\prime}+\left(\alpha^{2}+\omega^{2}\right) y=x
$$

Where $x$ is stress, $y$ is deformation, $\alpha$ is a damping ratio, $\omega$ is natural frequency, $\alpha^{2}+\omega^{2}$ is a "spring constant". Equation (1) is considered as a dynamic equation of a unit mass under action of external forces, a restoring force with the spring constant $\alpha^{2}+\omega^{2}$, and friction with coefficient of friction $2 \alpha$. The proposed approach is based on the desire to provide a compact

\footnotetext{
*Corresponding author: vitoz_1309@mail.ru
} 
solution to the equation. For the same purpose, we use a special time scale. The estimated duration of the cutting process at a given point is assumed as a unit of time.

The general solution to equation (1) can be expressed using a weight function:

$$
W(t)=\frac{1}{\omega} e^{-\alpha t} \sin \omega t
$$

The integral form of the weight function is:

$$
y(t)=\int_{0}^{t} W(s) x(t-s) d s .
$$

The weight function represents the solution to equation (1) with zero initial conditions and input in the form of $\delta$-function $x(t)=\delta(t)$. Equation (1) can also be considered as homogeneous with initial conditions $y(0)=0 ; y^{\prime}(0)=1$.

Besides the weight function, the equation (1) can be considered as a transient process and the solution to equation(1) under the input action $x$ can be in the form of a step function $x(t)=$ $\theta(t)$, where $\theta(t)=0$ at $t<0$ and $\theta(t)=1$ at $t \geq 0$.

Integral (2) shows that the transient process can be expressed as an integral of the weight function:

$$
y_{t r}(t)=\frac{1}{\alpha^{2}+\omega^{2}}\left[1-e^{-\alpha t}\left(\cos \omega t+\frac{\alpha}{\omega} \sin \omega t\right)\right] .
$$

The transient process shows that it reaches its maximum value at the moment $t=\frac{\pi}{\omega}$, which is equal to $\frac{1+e^{\alpha \frac{\pi}{\omega}}}{\alpha^{2}+\omega^{2}}$.

The limit value (steady-state value) of the transient process is:

$$
y_{\text {lim }}=\frac{1}{\alpha^{2}+\omega^{2}} \text {. }
$$

Figure 1 shows the weight function and the transient process of system (1) for the following parameters: $\alpha=0.02$ and $\omega=5$.

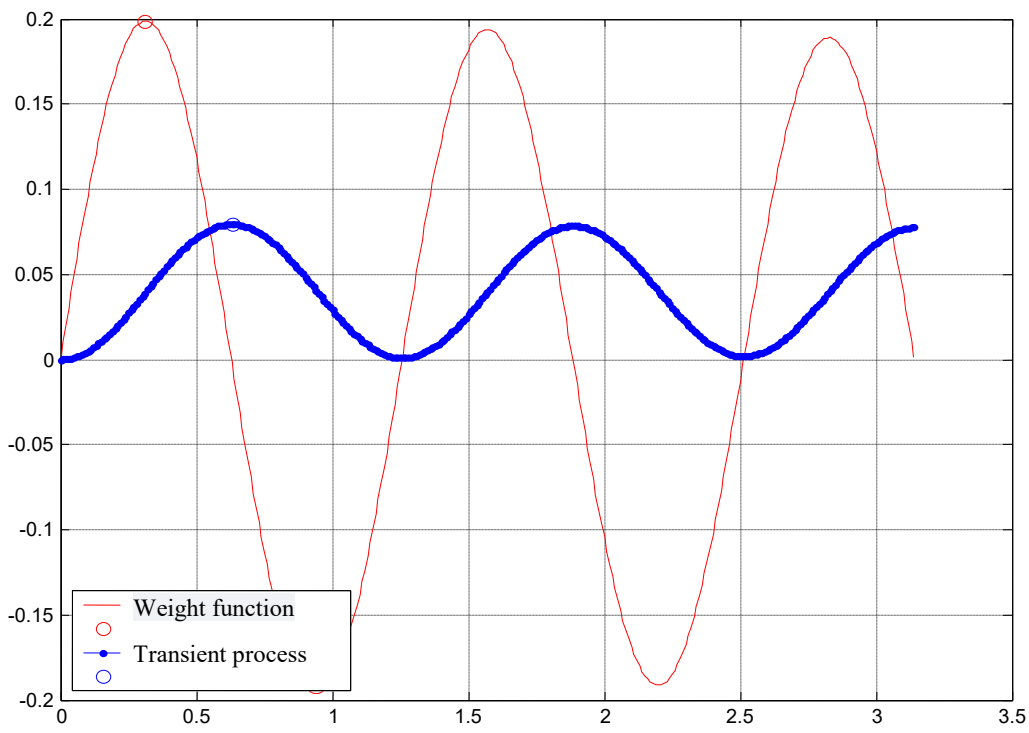

Fig. 1. The transient process of destruction of material. Weight function 
Low damping and significant values of natural frequency have been selected as typical parameters for the system.

In mechanics, the weight function and transient curves shown in Figure 1 represent the dependence of deformation of the cut material on time. In this case, the deformation is caused by a unit impulse and a unit step. Moreover, Figure 1 shows the points of maximum deformation. It is also seen that the use of an impulse instead of a step gives a greater maximum deformation and a higher rate of increase in deformation.

There is also another mechanical interpretation of the properties of the impulse transient function and the transient process in equation (1).

The equation describes the motion of a unit mass under a force with spring constant $\left(\alpha^{2}+\omega^{2}\right)$ and viscous friction with damping ratio $2 \alpha$.

If a point is under an impulse of unit intensity, that is $x(t)=\delta(t)$, where $\delta(t)$ is deltafunction, then according to the law of conservation of momentum $\Delta(m v)=F \Delta t$, where $m$ is mass, $v$ is velocity, and $F$ is a force acting over time $\Delta t$, unit mass changes the value of its own velocity by a unit vector.

If friction and restoring force are absent, the point will move at the obtained constant velocity. In the presence of a restoring force, as the point moves, the restoring force increases and, as a result of which, the point begins decelerating.

At a certain value of deformation, the velocity of the point turns to zero and then changes sign. At that moment, the point deviation (deformation in our case) reaches its maximum value and then begins to decrease to zero. Then the oscillation is repeated with the opposite sign. The friction additionally contributes to the decrease in velocity and leads to the oscillations being damped, so that the deformation reaches its maximum value only during the first oscillation.

If the input signal to the system $x(t)$ contains several impulses, then the reaction to the first impulse occurs in the described above way. At the moment of the second impulse, which is applied to the point until a coordinate of the point has taken a different sign, the point is given an additional velocity increment of the same sign, but less than from the initial one, since the impulse of the force is equal to the algebraic sum of the input impulse and impulses of restoring force and friction.

We can say that the efficiency of subsequent impulses is less than of the first one (the efficiency of an impulse is understood as a relative part of velocity increase caused by an impulse). With large deformation, the impulse efficiency can become zero. This fact explains the behavior of the transient process. Any continuous action on the system can be interpreted as a continuous sequence of impulses, since the efficiency of subsequent impulses decreases to zero, the point deviation or deformation maintains a steady-state constant value.

The obtained results have been confirmed by numerous studies of deformations at various profiles of applied loads. For a correct comparison of the effectiveness of different profiles, it is necessary to use their certain normalization. Let us assume that the total moment of the applied force is constant, which means that the integral of the force over time is constant, or, in other words, the area under the force graph is constant. Figures 2 and 3 show the results of modeling system (1) in the conditions of feeding it with rectangular impulses of width $\mathrm{h}$. For comparison, the graphs of the transient and weight functions are also shown.

Figures 2 and 3 reflect the behavior of the system under the same conditions, the only difference is that Fig. 2 shows the behavior of the system over a large time interval, and Fig. 3 over an interval slightly exceeding 1 . As the efficiency of the cutting process is affected by an increase in deformation over a short time interval, we assume that the cutting of material should occur during a time interval of less than 1 . 


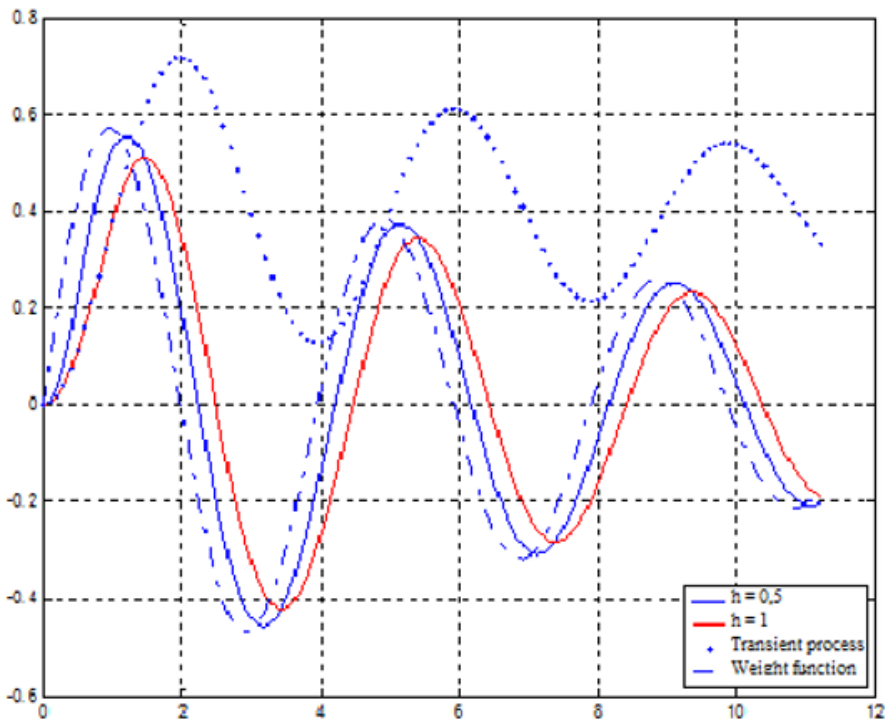

Fig. 2. Dependence of the destruction process on the duration of a jet action

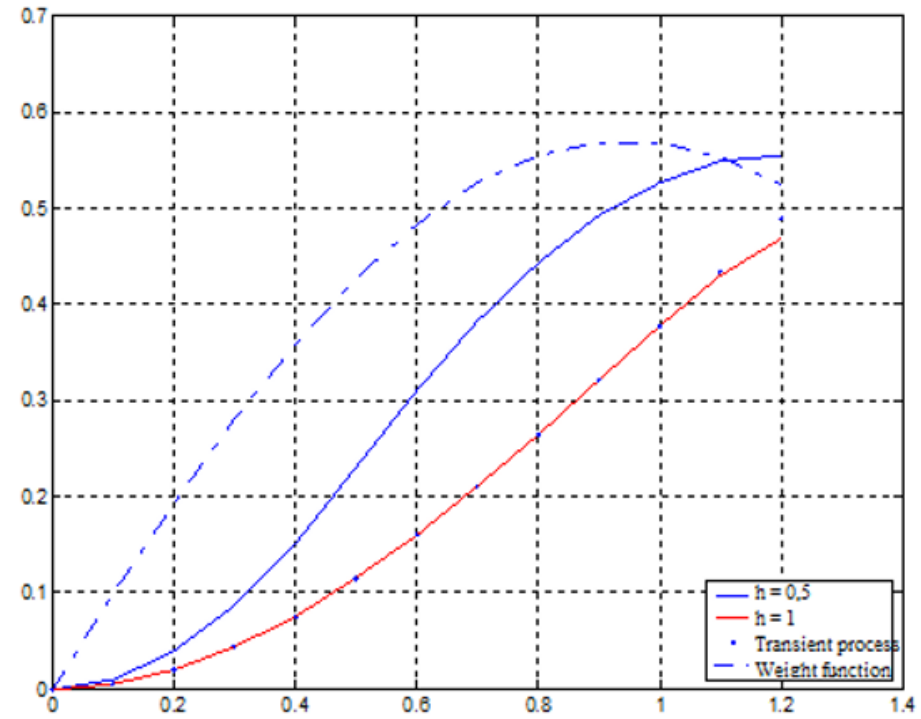

Fig. 3. Instant reaction of the system "material - jet"

Figures 2, 3 actually show the results of exposure to three types of impulses: with infinitely narrow width, with width $h=0,5$, and width $h=1$. As can be seen from the figures, the deformation increases with decreasing impulse width. The behavior of deformation at an impulse of width $h=1$ coincides with deformation at constant loading, which is not surprising since such an impulse coincides with a step action over a unit length.

When a jet of liquid is frozen several pieces of ice are formed along a unit length, thus, continuous loading is replaced by several impulses. The system has been simulated in a computer program calculating the deformation while loading the material with several identical impulses of total intensity equal to 1 and uniformly distributed over a unit segment. The calculation results are shown in Figure 4. 


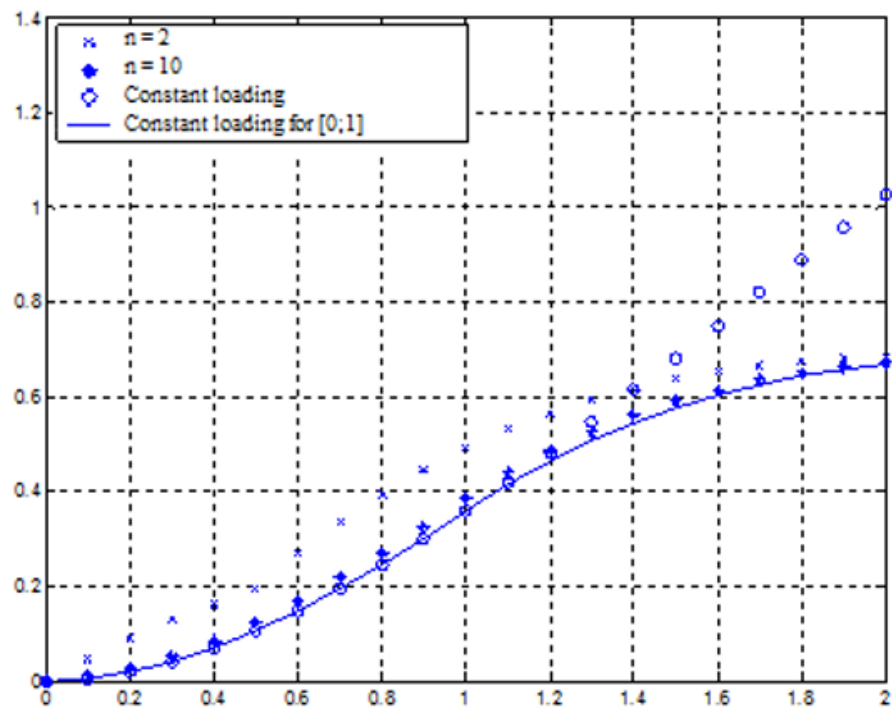

Fig. 4. Deformation changes for various forms of impulse

Figure 4 shows the dependence of deformation on time when two and ten impulses are applied, as well as when a continuous constant unit loading is applied on an infinite interval and on an interval of unit length.

The last two graphs at $0<t<1$ do not differ. Impulse loads lead to a greater deformation, and the deformation increases with a decrease in the number of impulses. This leads to the conclusion that freezing increases cutting efficiency by replacing continuous loading with impulses.

\section{Conclusion}

As can be seen, studies of the process of destruction of materials by a hypersonic ice water jet reach great attention. The article presents mathematical equations for determining the energy parameters, technological recommendations for the choice of treatment modes such as working pressure of a jet, diameter of nozzles, diameter of the feed channel for abrasive (ice), nozzle head feed, and distance from nozzles to the treated surface.

\section{References}

1. Yu.S. Stepanov, M.A. Burnashov, K.A. Golovin. Progressive technologies of hydrojet cutting of materials. (TulGU, Tula, 2009)

2. H. Kiyohashi, K. Handa. Proceedings of the International Symposium on New Applications of Waterjet Technology, Ishinomaki, Japan, pp. 51-60 (1999)

3. A.Y. Pushkarev, K.A. Golovin, A.B. Zabin, Y.I. Yeruhimivich, Proceeding of the International Symposium on New Applications of Water Jet Technology. Oktober 1921, Ishinomaki, Japan. Ishinomaki Senshu Universiti, pp. 421-425 (1999)

4. T.J. Louis, 5th American Waterjet Conference, Toronto, Canada, August, pp. 145 - 168 (1989)

5. Anon, High Pressure Water Jet Systems, Part 2, no. 4, pp. 20 -23 (1993) 